\title{
Blind Spot Detection Algorithm of Random Deployment WSN Research Based on Voronoi Diagram
}

\author{
Lijn $\mathrm{Gao}^{1}$, Hang Yin $^{1}$, Yuhua Wei $^{1 *}$, Le Wang ${ }^{1}$ \\ ${ }^{1}$ Department of Computer Science and Technology, ShenyangAerospaceUniversity, 110036, China \\ E-mail : wyh_weiyuhua@163.com
}

Key words: major disaster environment; WSN; blind spot detection; voronoi diagram

\begin{abstract}
In this paper we mainly research the coverage problem of WSN which is deployed by the UAV according to the pre-established track under the major disaster environment. Because the deployment regionis uneven and buildings may change at any time, the blind spot will be producein the deployment process.To overcome this problem we propose a blind spot detection method based on voronoi diagram.
\end{abstract}

\section{Introduction}

Wireless sensor networks (WSN) have become a hot research topic in recent years.WSN extends the ability to obtain information and connects the physical information of the objective world with the network together. WSN are widely used in military defense, industrial and agricultural control, urban management, bio-medical, environmental monitoring, disaster relief, remote control and other fields danger zone. It has attracted the great attention of the academia and industry in many countries and will produce great influence in the 21st century.

In order to accomplish the tasks of targets monitoring and acquiring information, WSN must ensure that the sensor node can effectively cover the target monitoring region.However, since the perception radius of sensor node is fixed, the random deployment of sensor nodes may produce blind spot, which will affect the network performance seriously.So the research of blind spot detection algorithm of WSN has important significance.

In this paper we mainly research the coverage problem of WSN which is deployed by the UAV according to the pre-established track under the major disaster environment. Because the deployment region is uneven and buildings may change at any time, the blind spot will be produce in the deployment process. To overcome this problem we propose a blind spot detection method based on voronoi diagram.

Here we review the existing blind spot detection algorithm of WSN.Ghrist R. [1] and Silva VD[2] used the homology theory of algebraic topology to detect the blind spot.Since the algorithm is a centralized algorithm with large calculation and not suitable for large-scale networks. If there are $n$ sensor nodes, the time complexity of the algorithm is $\mathrm{O}\left(\mathrm{n}^{5}\right)$. Buchart J. T. [3] established a communication connection diagram for WSN firstly, then using the biggest simplicial complex to transform the connection diagram into a two-dimensional simplicial complex, and based on the network model proposed a blind spot detection algorithm.CorkeP. [4] proposed a path density algorithm which detects blind spot according to the path density around the node. The correct rate of algorithm is high, but the energy consumption is large too. In view of previous research, we propose a blind spot detection algorithm based on the voronoi diagram.

\section{Blind Spot Detection Method of Random Deployment WSN Research Based on Voronoi Diagram}

Random deployment of the sensor node cannot cover the monitoring region completely.In addition, node failure, energy depletion and other reasons can also cause blind spot. Aiming at these problems, we propose a blind spot detection method based on the voronoi diagram in random deployment. In the detection method, sensor nodes are considered as a point set which is collected 
by the UAV. Then we createa voronoi diagram of the monitoring region according to the point set, and judge whether there are blind spots in the WSN.

A voronoi diagram is a way of dividing space into a number of regions. A set of points (called seeds, sites, or generators) is specified beforehand and for each seed there will be a corresponding region consisting of all points closer to that seed than to any other. The regions are called voronoi cells. It is dual to the delaunay triangulation. If there are n sensor nodes in a WSN, the voronoi diagram divides the space into $\mathrm{n}$ districts, each region include a point (a sensor node), the region is a point set, which distance is closer to the seed sensor node than the other.

We define the voronoi cell and Voronoi diagram as follows:

Definition 1. Consider P: $\left\{p_{1}, p_{2}, \ldots, p_{n}\right\}$ where $2 \leq$ nand $_{i} \neq p_{j}$ for $i \neq j, i, j \in \mathrm{I}_{\mathrm{n}}=1, \ldots \mathrm{n}$. The region given by $\mathrm{V} \mathrm{C}\left(p_{i}\right)=\mathrm{p} \mid \mathrm{d}\left(p, p_{i}\right)<=\left(p, p_{i}\right)$ where $\mathrm{d}\left(p, p_{i}\right)$ is the minimum euclidean distance between $\mathrm{p}$ and $\mathrm{p}_{\mathrm{i}}$ is called the voronoi cell (VC) associated with $p_{i}$.

Definition 2. The set of Vorono cells given b V D $(\mathrm{P})=\left\{\mathrm{VC}\left(p_{1}\right), \ldots, \mathrm{VC}\left(p_{n}\right)\right\}$ is called the voronoi diagram (VD) generated by $\mathrm{P}$.

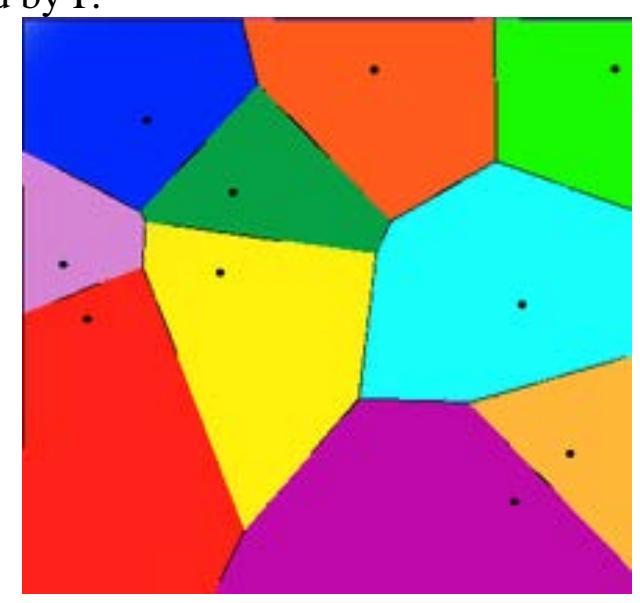

Fig. 1 Voronoi Figure

\section{Voronoi Diagram Generating Method}

Here we use the dual generation method to generate the voronoi diagram.The first step is generating delaunay triangulation, the second step is doing three vertical lines of each delaunay triangulation and forming the polygon mesh with each vertex of the delaunay triangulation as seed.

\subsection{GeneratingDelaunay Triangle Networking byInsertion Point-by-Point Method}

First, we use the UAV to collect the relative position coordinate of sensor node, and then established digital terrain model in the d-dimensional Euclidean space. Next we generate the delaunay triangle networking by insertion point-by-point method. The specific algorithm process as follows:

(1)Traverse all the scattered points, find the bounding box of the point set, obtain the initial triangle of the point set convex hull and put them in the triangle list.

(2)Insert the scattered point in turn,find out the triangle circumcircle which contains the new insertion point(called affected triangle by insertion point), delete the public sides of the affected triangle,then connect the insertion point with all the vertices of affected triangles.

(3)The new triangle will be optimized according to optimization criterion(such as interchangeable diagonal, etc.).

(4)Loop through the step 2 until all the scattered point insertion is completed.

From The algorithm, we can see that adding a new node need not rebuild the completed networking, just rebuild the affected triangle,so the build networking method is simple. Similarly, deletion or move a point can be done quickly and dynamically too. 


\subsection{Voronoi Polygon Generation Algorithm}

Voronoi polygon is special convex hull which only containsone point, the main building processis as follows:

(1)Starting from any point of the set, search for the shared vertex triangle in the Triangles_Collection (Triangles_Collection is the collection of delaunay triangle) to obtain their circumcenter coordinate and store in a array V_Point.

(2) Points in V_Point will be sort by using the algorithm of Graham Convex hull. And calculate the angle between horizontal line and the lines which generate by connecting this point with the other points. Then sort by angle size from small to big to obtain the ordered vertices set of voronoi polygon.

(3)Starting from the first point of the sorted set, connect these points in the order. We can obtain the voronoi polygon of this point. It is shown in dotted lines in Figure 1.

(4) Repeat step 1 to step 4 until all Voronoi polygons have been completed.
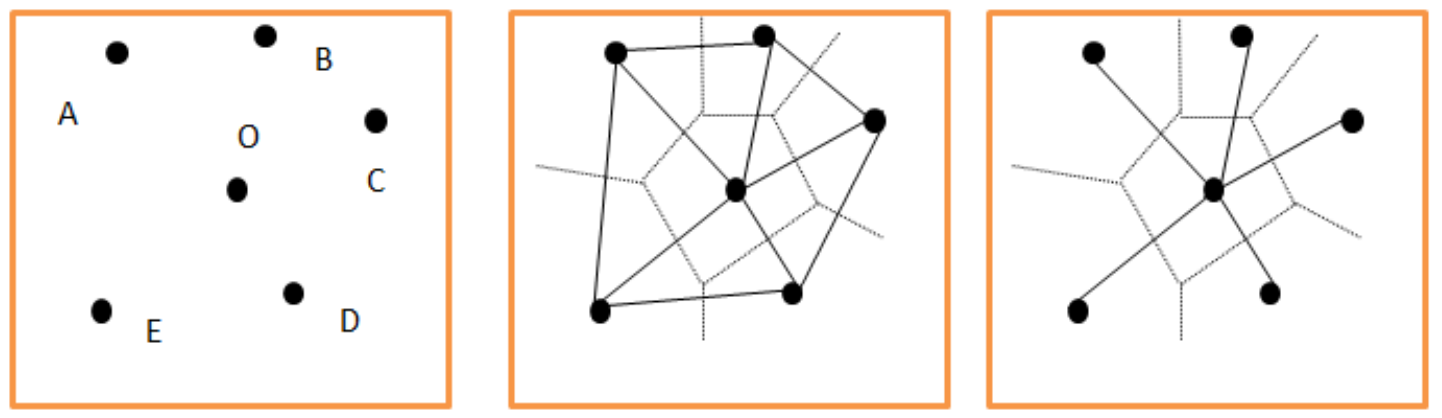

Fig. 2generate the voronoi diagram by dual generation method

\subsection{Blind spot detection Method based on Voronoi Diagram}

We assume that $d(p, v)$ denotes the distance between seed sensor node and its voronoi diagram vertex, where $P$ is the sensor, $v$ represents voronoi diagram vertices. Whether a monitoring region exists blind spot will be judged by d(p, v).

$$
d(p, v)=\sqrt{\left(p_{x}-v_{x}\right)^{2}+\left(p_{y}-v_{y}\right)^{2}}
$$

We assume that $R_{S}$ denotes the sensing range of sensor. As shown in figure 1 , if the distance between seed sensor node $\mathrm{O}$ and its voronoi diagram vertices $(\mathrm{A}, \mathrm{B}, \mathrm{C}, \mathrm{D}, \mathrm{E})$ is less than $R_{s}$, there is no blind spot in its voronoi diagram region; If the distance between seed sensor node $\mathrm{O}$ and its voronoi diagram vertices $(\mathrm{A}, \mathrm{B}, \mathrm{C}, \mathrm{D}, \mathrm{E})$ is great than $R_{s}$, there exist blind spot in its Voronoi diagram region.

\section{Conclusion}

In this paper we mainly research the coverage problem of WSN which is deployed by the UAV according to the pre-established track under the major disaster environment. And propose a blind spot detection method based on voronoi diagram. But the problem of the blind spots restoration method does not fully discussed, further research is needed.

\section{Acknowledgment}

This work was partially supported by the Natural Science Foundation of Liaoning Province under grant No.2013024020 and No.2013024001.Thank AVIC Technology Innovation Fund to support, fund number: 2013S60109R. 


\section{References}

[1]R. Ghrist, A. Muhammad, "Coverage and hole-detection in sensor networks via homology," Proceedings of the 4th international symposium on Information processing in sensor networks, April 2005 , pp. 254-260.

[2]De Silva V, R. Ghrist, “Homological sensor networks,” Notices of the American mathematical society, Vol. 54,No. 1, 2007.

[3] J. Kanno, J. G. Buchart, R. R. Selmic, "Detecting coverage holes in wireless sensor networks,” Proceedings of the 4th international symposium on Information processing in sensor networks , 2009, pp. 24-26.

[4] P. Corke, R. Peterson, D. Rus, "Finding holes in sensor networks,” the IEEE Workshop on Omniscient Space: Robot Control, 2007, pp. 1-8. 